\title{
Centrality dependence of the multiplicity and transverse momentum distributions at RHIC and LHC and the percolation of strings
}

\author{
M. A. Braun ${ }^{\mathrm{a}}$, F. del Moral ${ }^{\mathrm{b}}, \underline{\text { C. Pajares }^{\mathrm{c}}}$ \\ aDepartment of High Energy Physics, University of St. Petersburg, \\ 19894 St. Petersburg, Russia

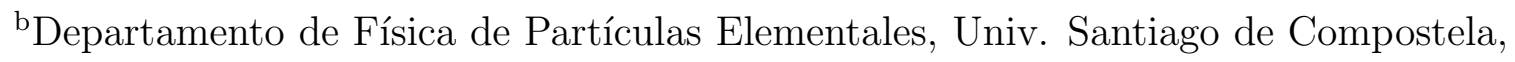 \\ 15706 Santiago de Compostela, Spain
}

The dependence of the multiplicity and the transverse momentum distribution on the number of collisions are studied for central and peripheral $\mathrm{Au}-\mathrm{Au}$ collisions at SPS, RHIC and LHC energies in the framework of percolation of strings. A scaling law relating the multiplicity to the mean transverse momentum is obtained. Our results are in overall agreement with the SPS and RHIC data, obtaining a suppression on $p_{T}$ distribution even for $p_{T}$ larger than $1 \mathrm{GeV} / \mathrm{c}$.

\section{INTRODUCTION}

Most of the multiparticle production is currently described in terms of color strings stretched between the projectile and target, which decay into new strings and subsequently hadronize to produce observed hadrons. Color strings may be viewed as small areas in the transverse space, $\pi r_{o}^{2}, r_{o}=0.2 \mathrm{fm}$, filled with color field created by the colliding partons. Particles are produced via emission of $q \bar{q}$ pairs in this field [1]. With growing energy and/or atomic number, the number of strings grows, and they start to overlap, forming clusters, very much similar to disks in the two dimensional percolation theory [2]. At a certain critical density,

$$
\eta_{c}=N_{s} \pi r_{o}^{2} / S
$$

a macroscopic cluster appears that marks the percolation phase transition. In (臬) $N_{s}$ is the number of strings and $S$ is the total transverse area of the scattering of the colliding nuclei.

The percolation theory governs the geometrical pattern of the string clustering. Its observable implications, however, require introduction of some dynamics to describe string interaction, i.e., the behavior of a cluster formed by several overlapping strings. There are several possibilities [3, 4]. Here, we assume that a cluster behaves as a single string with a higher color field $\vec{Q}_{n}$ corresponding to the vectorial sum of the color charge of each individual $\vec{Q}_{1}$ string. The resulting color field covers the area $S_{n}$ of the cluster. As $\vec{Q}_{n}=\sum \vec{Q}_{1}$, and the individual string colors may be oriented in an arbitrary manner respective to one another, the average $\vec{Q}_{1 i} \vec{Q}_{1 j}$ is zero and $\vec{Q}_{n}^{2}=n \vec{Q}_{1}^{2}$. 
Knowing this charge color $\vec{Q}_{n}$, one can compute the particle spectra produced by a single color string of area $S_{n}$ using the Schwinger formula [5]. For the multiplicity $\mu_{n}$ and the average $p_{T}^{2}$ of particles $\left\langle p_{T}^{2}>_{n}\right.$ produced by a cluster of $n$ strings one finds

$$
\mu_{n}=\sqrt{\frac{n S_{n}}{S_{1}}} \mu_{1} \quad ; \quad<p_{T}^{2}>_{n}=\sqrt{\frac{n S_{1}}{S_{n}}}<p_{T}^{2}>_{1}
$$

where $\mu_{1}$ and $<p_{T}^{2}>_{1}$ are the mean multiplicity and $p_{T}^{2}$ of particles produced by a simple string with area $S_{1}=\pi r_{o}^{2}$. For strings, just touching each other $S_{n}=n S_{1}$ and hence $\mu_{n}=n \mu_{1},<p_{T}^{2}>_{n}=<p_{T}^{2}>_{1}$, as expected. In the opposite case of maximum overlapping $S_{n}=S_{1}$ and therefore $\mu_{n}=\sqrt{n} \mu_{1},<p_{T}^{2}>=\sqrt{n}<p_{T}^{2}>_{1}$

Equation (2) is the main tool of our calculation [6]. In order to compute the multiplicities we generate strings according the Monte Carlo code of [1]. Each string is produced at an identified impact parameter. From this, knowing the transverse area of each string, we identify all the clusters formed in each collision and subsequently compute for each of them its multiplicity in units $\mu_{1}$. The value of $\mu_{1}$ was fixed by normalizing our results to the SPS WA98 results for central $\mathrm{Pb}-\mathrm{Pb}$ collisions [7]. In color strings models both $\mu_{1}$ and $<p_{T}^{2}>$ are assumed to rise with energy due to increase of the rapidity interval available and to the hard contribution. However this rise is very weak in the range $\sqrt{s}=17-200$ $\mathrm{GeV}$ and we can neglect it, in the first approximation.

\section{RESULTS}

The comparison of our results for the dependence of the multiplicity on the number of participants with the SPS WA98 data, the RHIC Phenix [8] and Phobos [9] data at $\sqrt{s}=130 \mathrm{GeV}$ and $\sqrt{s}=200 \mathrm{GeV}$, is presented in Fig. 1. a, where also is presented our prediction for LHC.

In order to compute the transverse momentum distribution we make use of the parametrization of the $p p$ data at $130 \mathrm{GeV}$

$$
\frac{d N}{d p_{T}^{2}}=\frac{a}{\left(p_{o}+p_{T}\right)^{\alpha}}
$$

where $a, p_{o}$ and $\alpha$ are parameters fitted to data. The standardly expected distribution for $\mathrm{Au}-\mathrm{Au}$ collision is

$$
\frac{d N}{d p_{T}^{2}}=<N_{\text {coll }}>\left.\frac{d N}{d p_{T}^{2}}\right|_{p p}
$$

However, from Eq. (1) we have

$$
<p_{T}^{2}>_{A u-A u}=<p_{T}^{2}>_{p p} \frac{<n S_{1} / S_{n}>_{A u-A u}^{1 / 2}}{<n S_{1} / S_{n}>_{p p}^{1 / 2}}
$$

This implies that the same parametrization (3) can be used for nucleus-nucleus collisions with the only change

$$
p_{o} \rightarrow p_{o}\left(\frac{<n S_{1} / S_{n}>_{A u-A u}}{<n S_{1} / S_{n}>_{p p}}\right)^{1 / 4}
$$



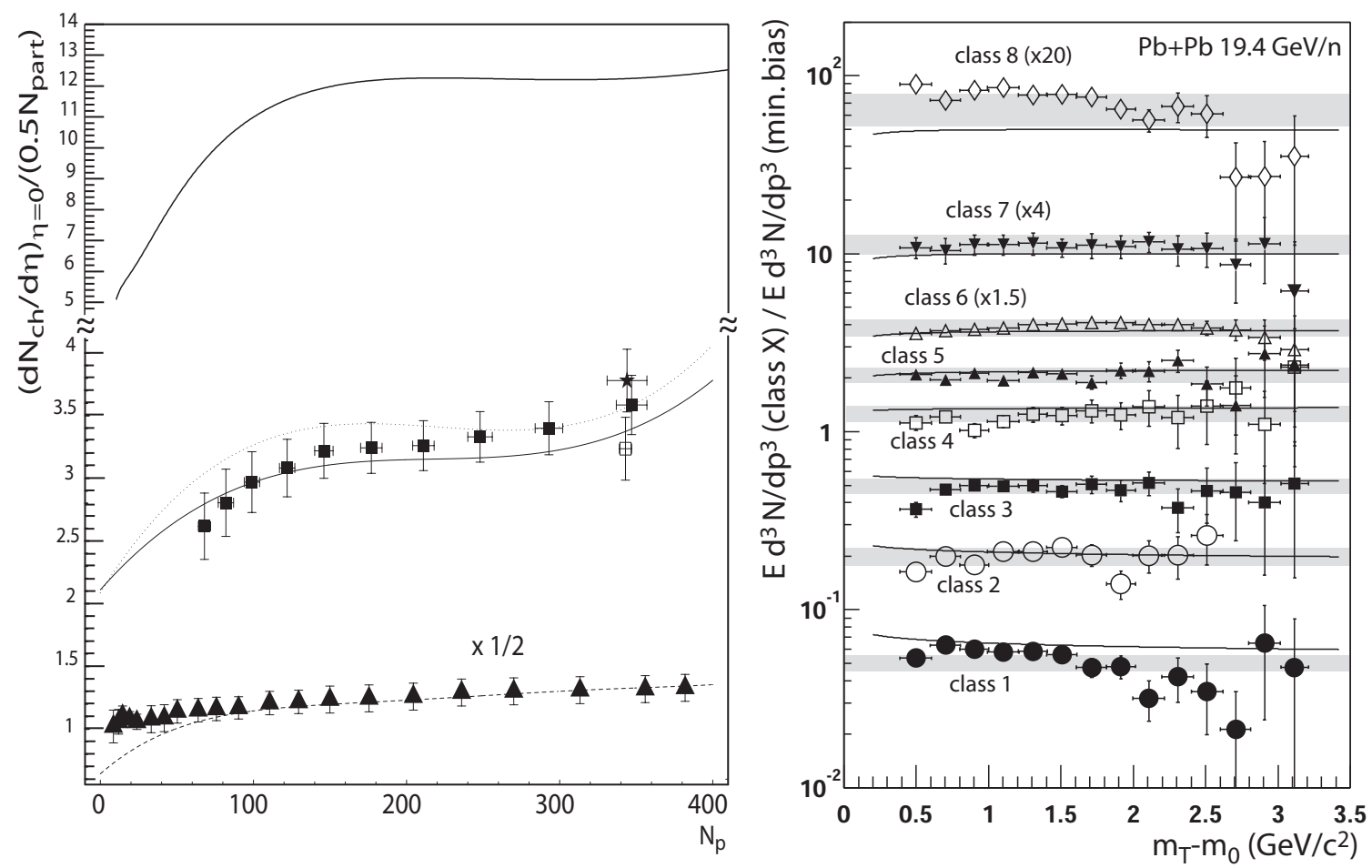

Figure 1. a) Comparison of our predictions of the multiplicity with the number of participants, the SPS WA98 [7] (filled triangles), the RHIC Phenix [8] (filled boxes), Phobos [9] (nonfilled box) data at $\sqrt{s}=130 \mathrm{GeV} / \mathrm{n}$, and RHIC Phenix data at $\sqrt{s}=200 \mathrm{GeV} / \mathrm{n}$ (filled star). The upper solid line represents our prediction for $\mathrm{Pb}-\mathrm{Pb}$ collisions at $5500 \mathrm{GeV} / \mathrm{n}$. b) Comparison of our predictions of ratios of invariant multiplicities of neutral pions for $\mathrm{Pb}-\mathrm{Pb}$ collisions of different centralities to minimum bias distributions as a function of $m_{T}-m_{o}$ and SPS WA98 data. Meaning of centrality classes and grey bands can be found en reference [11].

In Fig. 1.b we show the comparison of our results for eight different centrality events in $\mathrm{Pb}-\mathrm{Pb}$ collisions with WA98 data [11]. In Fig. 2. a we show the distribution (3) for charged particles for central (5\%) Au-Au collisions compared to the Phenix data [10]. Also the distribution expected from the independent string picture, Eq. (荬), is shown. A very good agreement is obtained. We also obtain a good agreement for peripheral collisions, see reference [6]. In Fig. 22.b we show our prediction for central Au-Au collisions at $\sqrt{s}=5500 \mathrm{GeV}$ compared with the formula (4). In this case, contrary to RHIC energy, a crossing at low $p_{T}$ is predicted.

We observe that for a cluster, $<p_{T}^{2}>=\frac{S_{1}}{S_{n}} \frac{<p_{T}^{2}>1}{\mu_{1}} \mu_{n}$, which at high density translate into

$$
<p_{T}^{2}>_{A A}=\frac{\pi r_{o}^{2}}{S_{A A}} \frac{<p_{T}^{2}>_{1}}{\mu_{1}} \mu_{A A}
$$

The formula (7) shows an universal relation between multiplicities and $<p_{T}^{2}>$. The scaling laws expressed by the formula (3) with the change (6) and by the formula (7) are 

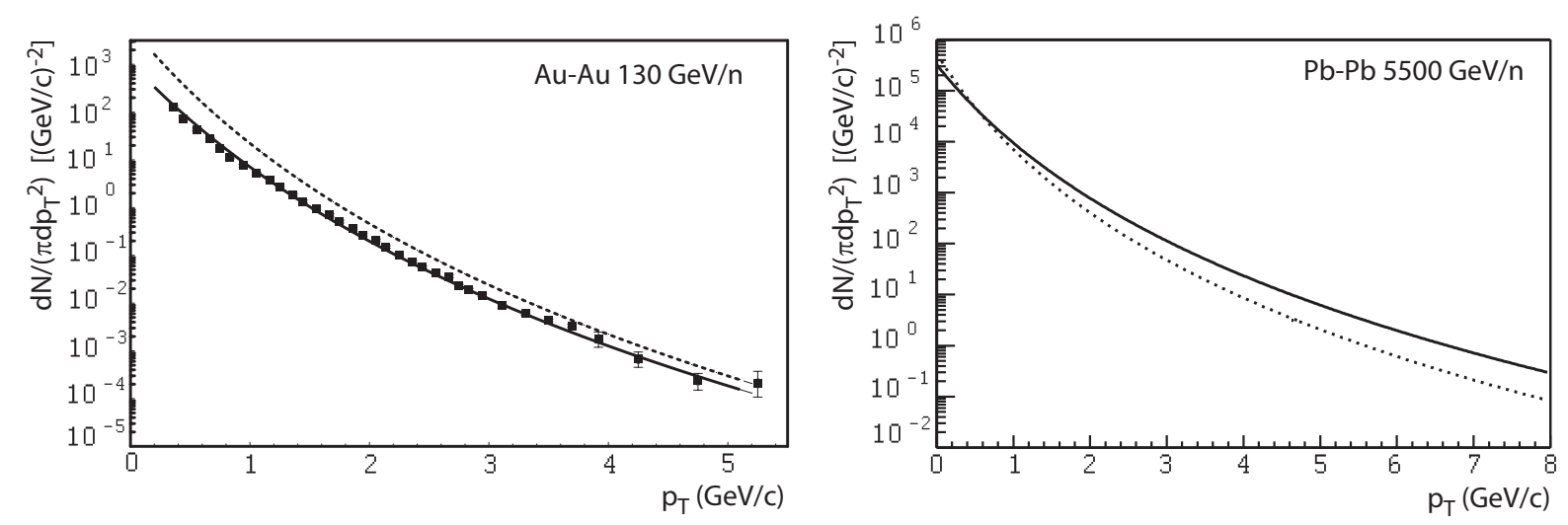

Figure 2. Expected $p_{T}$ distribution using the percolation of strings model (solid line) for $5 \%$ central $\mathrm{Au}-\mathrm{Au}$ and $\mathrm{Pb}-\mathrm{Pb}$ collisions at $130 \mathrm{GeV} / \mathrm{n}$ and $5500 \mathrm{GeV} / \mathrm{n}$ respectively. Also it is shown the expected distribution from the independent string picture (dotted line).

very similar to other scaling laws using saturation of gluons.

To conclude, a general comment. We obtain a good description of the multiplicities and transverse momentum distribution at different energies and different centralities including a low and high $p_{T}$ suppression. However we can not reproduce the additional high $p_{T}$ suppression reported at this conference by several collaborations. If the explanation is jet quenching, our approach can provide an unified framework to add the phenomenology of jet quenching. This work was done under contract FPA2002-01161 of Ministerio de Ciencia y Tecnologia of Spain.

\section{REFERENCES}

1. N. S. Amelin, M. A. Braun and C. Pajares, Z. Phys. C 63 (1994) 507; N. S. Amelin, N. Armesto, C. Pajares and D. Sousa, Eur. Phys. J. C 22 (2001) 149; N. Armesto, C. Pajares and D. Sousa, Phys. Lett. B 527 (2002) 92.

2. N. Armesto, M. A. Braun, E. G. Ferreiro and C. Pajares, Phys. Rev. Lett. 77 (1996) 3736; M. Nardi and H. Satz, Phys. Lett. B 442 (1998) 14.

3. M. A. Braun, C. Pajares and J. Ranft, J. Mod. Phys. 14 (1998) 2689; M. A. Braun and C. Pajares, Eur. Phys. J. C 16 (2000) 349.

4. M. A. Braun and C. Pajares, Phys. Rev. Lett. 85 (2001) 4864; M. A. Braun, F. del Moral and C. Pajares, Eur. Phys. J. C 21 (2001) 557.

5. J. Schwinger, Phys. Rev. 82 (1951) 664.

6. M. A. Braun, F. del Moral and C. Pajares, Phys. Rev. C 65 (2002) 024907.

7. M. M. Aggarwal et al., WA98 Collaboration, Eur. Phys. J. C 18 (2001) 651.

8. K. Adcox et al., Phenix Collaboration, Phys. Rev. Lett. 86 (2001) 3500.

9. B. B. Bock et al., Phobos Collaboration, Phys. Rev. Lett. 85 (2000) 3100.

10. K. Adcox et al., Phenix Collaboration, Phys. Rev. Lett. 88 (2002) 022301.

11. M. M. Aggarwal et al., WA98 Collaboration, Eur. Phys. J. C 23 (2002) 225. 